\section{Antibiotische Therapie bei Asthma-Exazerbation: Auswirkungen auf Verlauf?}

Stefan MS et al. Association of Antibiotic Treatment With Outcomes in Patients Hospitalized for an Asthma Exacerbation Treated With Systemic Corticosteroids. JAMA Intern Med 2019; 179: $333-339$

Obwohl die Leitlinien verschiedener Fachgesellschaften von der empirischen Behandlung mit Antibiotika bei exazerbiertem Asthma abraten, werden in den USA wie auch in vielen anderen Ländern hohe Verschreibungsraten verzeichnet. Die vorliegende Studie untersuchte die Auswirkungen einer antibiotischen Therapie auf den Krankheitsverlauf bei Patienten/ innen, die aufgrund einer Asthma-Erkrankung stationär behandelt wurden und Glukokortikoide erhielten.

Die retrospektive Kohortenstudie verwendete Daten von 19811 Erwachsenen, welche aufgrund einer Exazerbation eines Asthmas unter anderem systemisch mit Glukokortikoiden behandelt wurden. Beteiligt waren 542 US-amerikanische Akutkliniken zwischen 2015 und 2016. Untersucht wurde eine frühe antibiotische Behandlung mit Beginn während der ersten beiden Tage und über mindestens 2 Tage des stationären Aufenthaltes. Primärer Endpunkt war die Krankenhausverweildauer. Sekundäre Endpunkte waren u.a. Therapieversagen (Einleitung einer mechanischen Beatmung, Verlegung auf eine Intensivstation nach Tag 2 der Krankenhausaufnahme, Mortalität während des stationären Aufenthaltes, Wiederaufnahme wegen Asthma innerhalb von 30 Tagen nach Entlassung), Behandlungskosten und therapiebedingte Nebenwirkungen wie allergische Reaktionen oder Diarrhoe. Ausgeschlossen wurden Patientinnen oder Patienten mit einer potenziellen Indikation für eine antibiotische Therapie, z.B. bei akuter oder chronischer Bronchitis, chronischobstruktiver Lungenerkrankung, Emphy- sem, Bronchiektasen, Sinusitis, Sepsis, Pneumonie, Harnwegsinfekt, Haut- oder Weichteilinfektionen als Zweitdiagnose oder positiver Blut- oder Sputumkultur bei Aufnahme. Zudem wurden Betroffene, welche von einer anderen Klinik zuverlegt oder an eine andere Klinik weiterverlegt worden waren, ebenfalls ausgeschlossen.

Das mediane Alter der Teilnehmenden war 46 Jahre (Interquartilsabstand [IQR] $34-59), 72,6 \%$ waren weiblich, $44,3 \%$ waren Weiße. 44,4\% aller Beteiligten erhielten eine antibiotische Therapie. Verglichen mit denjenigen ohne antibiotische Therapie waren erstere

- älter (im Median 48 [IQR 36-61] vs. 45 [IQR 32 - 57] Jahre),

- eher weißer Hautfarbe (48,6\% vs. $40,9 \%)$,

- eher Raucher (6,6\% vs. 5,3\%).

Außerdem hatten sie

- eine höhere Anzahl von Komorbiditäten (bspw. kongestive Herzinsuffizienz $6,2 \%$ vs. 5,8\%),

- eine signifikant längere Krankenhausverweildauer (Median 4 [IQR 3-5] vs. 3 [IQR 2 - 4] Tage),

- eine vergleichbare Rate an Therapieversagen $(5,4 \%$ vs. $5,8 \%)$ und

- höhere Behandlungskosten (Median 4911 US\$ [IQR $3283-7478$ ] vs. 3537 US\$ [IQR 2251 - 5789]).

Am häufigsten verordnet wurden Makrolide (51,9\%), Chinolone (34,8\%) und Cephalosporine der dritten Generation (19,6\%). Bei 134 Patientinnen und Patienten (1,5\%) wurde eine Antibiotikaassoziierte Diarrhoe diagnostiziert, in der Gruppe ohne antibiotische Therapie trat bei 91 (0,8\%) eine Diarrhoe auf.

Die Ergebnisse reflektieren, so Stefan et al., die Erfahrung bei unselektioniertem Patientengut und Behandlung unter Routine-Bedingungen und unterstützen die aktuellen Leitlinien, welche den Einsatz einer antibiotischen Therapie nur bei Nachweis entsprechender Infektionen empfehlen. Einschränkend geltend gemacht wird u.a., dass eine Kausalität der Zusammenhänge aufgrund der beobachtenden Natur der Studie nicht aufgezeigt werden kann.

\section{FAZIT}

In der vorliegenden retrospektivbeobachtenden Untersuchung war die empirische antibiotische Behandlung bei Asthma-Exazerbation assoziiert mit einer längeren Krankenhausverweildauer, höheren Behandlungskosten und vergleichbarem Risiko eines Therapieversagens. Dies unterstreicht, so Stefan et al., die Notwendigkeit, bei stationären AsthmaPatientinnen und Patienten eine antibiotische Therapie zu vermeiden, soweit die Indikation nicht eindeutig gesichert werden kann.

\section{Dr. Birgit Gappa, Penzberg}

\section{Kommentar}

In einer retrospektiven Metanalyse untersuchten Stefan et al. den Nutzen einer empirischen Antibiotika-Therapie bei Patienten mit instabilem und exazerbiertem Asthma während der ersten beiden Krankenhaustage. 19811 Patienten wurden in Hinblick auf Aufenthaltsdauer, Gesamtüberleben und Therapieansprechen untersucht. Verglichen mit der Vergleichskohorte ohne Antibiose, waren die Patienten älter, häufiger aktive Raucher und hatten mehr Komorbiditäten. In Bezug auf das Therapieansprechen der Grunderkrankung gab es keine Unterschiede. Der nicht gezielte Einsatz einer Antibiose bei Asthmapatienten im frühen, stationären Umfeld führte in dieser Studienübersicht nicht zu einem belegbaren Nutzen. Die Autoren schlussfolgern, dass eine kalkulierte Antibiose nur bei gesichertem Hinweis auf eine Pneumonie (CAP) bzw. einem mit SymptomScore belegten Status febrilis bei Patienten mit Asthma und stationärer Therapienotwendigkeit eingesetzt werden sollte. 
Kritisch zu bemerken ist, dass die retrospektive, auf einer „propensity score matchin analysis“ basierende Untersuchung nicht sicher die Diagnosezuordnung belegen lässt und dass der sinnvolle Einsatz bei belegbarem Verdacht auf eine Pneumonie bei diesem Patientengut mit obstruktiven Atemwegserkrankungen nicht zu hinterfragen ist.

Autorinnen/Autoren

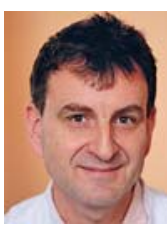

Prof. Dr. med.

Christian Grohé,

Klinik für Pneumologie,

Evangelische Lungenklinik Berlin

christian.grohe@pgdiako-

nie.de 